\title{
M1a Stage Finding
}

National Cancer Institute

\section{Source}

National Cancer Institute. M1a Stage Finding. NCI Thesaurus. Code C48701.

A TNM finding indicating the spread of cancer to distant anatomic sites. The definition of M1a TNM finding depends on the specific type of cancer that it refers to; for example, for colorectal cancer it refers to metastasis confined to one organ or site (e.g., liver, lung, ovary, nonregional node); for prostate cancer it refers to metastasis to non-regional lymph node(s); for bone cancer it refers to metastasis to the lung. 